\title{
Transferrin receptor 1: a target for antibody-mediated cancer therapy
}

\author{
“... studies in animal models and initial clinical trials suggest \\ that certain antibody-based cancer therapeutics will be \\ effective in humans."
}

First draft submitted: 8 April 2016; Accepted for publication: 9 May 2016; Published online: 4 July 2016

Keywords: antibody effector functions • cancer immunotherapy • CD71 • cytotoxic antibody - drug delivery • iron deprivation $\bullet$ receptor-mediated endocytosis $\bullet$ transferrin receptor 1

The transferrin receptor 1 (TfR1), also known as CD71, is a homodimeric protein that is a key regulator of cellular iron homeostasis and proliferation [1,2]. It is a type II transmembrane protein with a large extracellular domain. This receptor interacts with iron-loaded transferrin (Tf) to import iron into the cell. The TfR1/Tf/iron complex is taken up through constitutive clathrin-mediated endocytosis. Due to the decrease in $\mathrm{pH}$ in intracellular vesicles, iron is released from Tf and the TfR1/Tf complex is recycled back to the cell surface where Tf dissociates from the receptor. Iron is a cofactor of intracellular enzymes including the ribonucleotide reductase that is coupled to DNA synthesis and, thus, required for cellular proliferation. Therefore, although TfR1 is expressed at a low level in a broad variety of cells, it is expressed at higher levels in rapidly proliferating cells, including malignant cells in which overexpression has been associated with poor prognosis [1,2]. Interestingly, TfR1 has also been shown to mediate NF- $\kappa \mathrm{B}$ signaling in malignant cells through the interaction with the inhibitor of the $N F-\kappa B$ kinase (IKK) complex, increasing cancer cell survival [3]. Moreover, TfR1 can contribute to mitochondrial respiration and the production of reactive oxygen species (ROS), which play pivotal roles in tumor growth [4]. TfR1 overexpression on malignant cells and its central role in cancer cell pathology make it a meaningful target for antibody-mediated cancer therapy.
Depending on the position of the targeted TfR1 site (epitope), antibodies can be neutralizing, meaning that they inhibit Tf binding, or they can be non-neutralizing. The TfR1 can be targeted for two nonmutually exclusive purposes in cancer therapy. First, receptor internalization is attractive for the purpose of drug delivery. Therapeutic agents coupled to either an antibody or the ligand Tf may be internalized into cancer cells. This strategy has been extensively used for the delivery of a variety of agents including chemotherapeutic drugs, toxins, genes, oligonucleotides, small inhibitory RNA (siRNA), enzymes, viral vectors and nanodrugs [1]. Compared to Tf, anti-TfR1 antibodies have several advantages: compared to antibodies that do not cross-react with TfR2, another version of the receptor mostly expressed in hepatocytes, Tf will target both TfR1 and TfR2, potentially leading to more liver toxicity; Tf in the blood may block the binding of Tf conjugates to TfR1, which may potentially decrease the therapeutic efficacy. Although, this may also be the case for neutralizing antibodies that share the Tf binding site, it would not be an issue for non-neutralizing antibodies. The second purpose for TfR1 targeting is to use antibodies as direct cytotoxic agents that disrupt the function of the receptor through inhibition of Tf binding, inhibition of TfR1 internalization, or disruption of receptor cycling due to its intracellular sequestration and degradation; all of
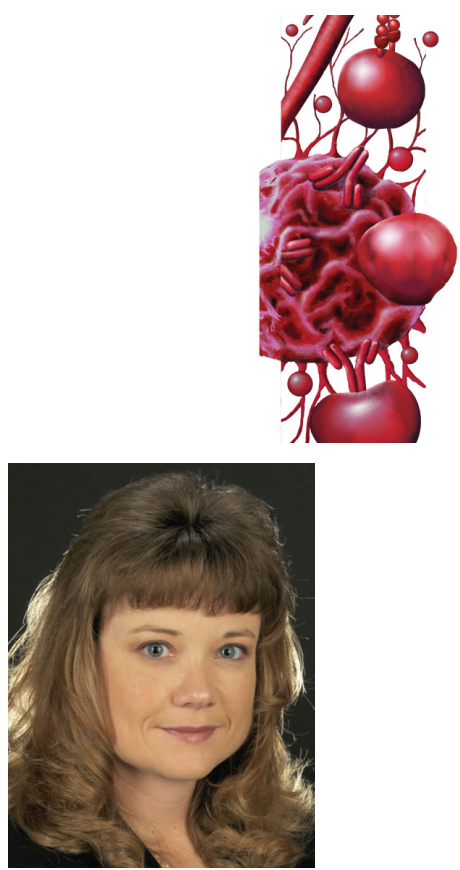

Tracy R Daniels-Wells Division of Surgical Oncology, Department of Surgery, David Geffen School of Medicine at UCLA, Los Angeles, CA, USA

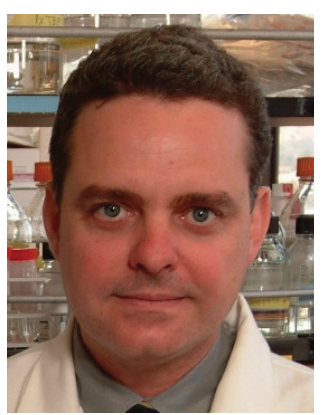

Manuel L Penichet ${ }^{1,2,3,4,5}$

'Division of Surgical Oncology, Department of Surgery, David Geffen School of Medicine at UCLA, Los Angeles, 10833 Le Conte Ave, CHS 54-140, Box 951782, Los Angeles, CA 90095-1782, USA

2Department of Microbiology, Immunology, \& Molecular Genetics, David Geffen School of Medicine at UCLA, Los Angeles, CA, USA

${ }^{3}$ The Molecular Biology Institute, UCLA, Los Angeles, CA, USA

${ }^{4} J o n s s o n$ Comprehensive Cancer Center, UCLA, Los Angeles, CA, USA

${ }^{5}$ UCLA AIDS Institute, CA, USA

*Author for correspondence:

Tel.: +1 3108251304

Fax: +13108257575

penichet@mednet.ucla.edu

Future $\because$

Medicine part of 
which ultimately interfere with iron uptake leading to lethal iron starvation [2]. Additionally, both neutralizing and non-neutralizing antibodies can be potentially cytotoxic through the induction of $\mathrm{Fc}$ effector functions: antibody-dependent cell-mediated cytotoxicity (ADCC), antibody-dependent cell-mediated phagocytosis (ADCP), and complement-dependent cytotoxicity (CDC) [2,5].

The number of agents that have been delivered into cancer cells by anti-TfR1 antibodies is too numerous to be mentioned here [1]. As an example, we have developed a mouse/human chimeric IgG3 antibodyavidin fusion protein specific for human TfR1 [6,7]. This molecule (ch128.1Av) has the variable regions of the murine antibody 128.1, does not cross react with TfR2, does not inhibit Tf binding to TfR1, and is dimeric in solution due to the tetrameric structure of avidin [6,8]. ch128.1Av has been shown to deliver biotinylated saporin (a plant toxin) and a biotinylated lentiviral vector containing a therapeutic gene into malignant $\mathrm{B}$ cells in vitro [9-11]. The cancer targeting potential of the lentiviral vector was further enhanced through the use of an immunoglobulin promoter that limits the expression of the therapeutic gene to B cells. Since tumor targeting occurs at two levels, the potential of unwanted side effects is reduced with this conjugate. We have also shown that the parental antibody without avidin (ch128.1) can deliver modified lentiviruses expressing the immunoglobulin binding domain of protein A on the surface of the virus [11]. Another relevant example is a fully human anti-human TfR1 IgG1 antibody labeled with a radionucleotide that demonstrated antitumor activity in a xenograft mouse model of pancreatic cancer [12].

Clinical trials have been conducted with anti-TfR1 antibodies for delivery purposes since the 1990s. An immunotoxin composed of 454A12, a murine IgG1 that targets the human TfR1, conjugated to the catalytic subunit of the plant toxin ricin (RTA) was evaluated for antitumor efficacy in two clinical trials $[13,14]$. Preliminary results were reported for one trial in which local treatment of metastatic intraperitoneal carcinoma showed no antitumor effect and systemic toxicity was observed including a fatal encephalopathy with cerebral edema [13]. In the other study, patients with leptomeningeal systemic neoplasia that received local intraventricular treatment with 454A12-RTA demonstrated greater than a $50 \%$ reduction of tumor cell counts in the cerebrospinal fluid and no systemic toxicity was detected [14]. These trials suggest that antiTfR1 immunotoxins may have efficacy when administered locally in certain cases; however, toxicity is a concern, particularly in the case of systemic administration. This problem may be overcome by changing the delivered cargo. Immunoliposomes coated with a single chain $\mathrm{Fv}(\mathrm{scFv})$ specific for TfR1 encapsulating plasmid DNA encoding the wild-type $p 53$ gene (known as SGT-53) have been evaluated in a clinical trial [15]. p53 is a tumor suppressor protein whose function is lost in multiple malignancies. The first Phase I clinical trial using this agent showed that $\mathrm{p} 53$ accumulated in metastatic lesions of several types of advanced solid tumors, SGT-53 was well tolerated, and most patients demonstrated stable disease. Since restoration of p 53 function would sensitize the malignant cells to cytotoxic drugs, a Phase I clinical trial (NCT02354547) and two Phase II clinical trials (NCT02340117 and NCT02340156) are currently in progress using SGT53 combined with chemotherapeutic drugs.

As mentioned above, anti-TfR1 antibodies can also be used as direct anticancer agents. Extensive cross-linking of the receptor results in cytotoxicity. For example, the rat anti-mouse TfR1 IgM antibody R17 208 does not block Tf binding; however, it blocks the internalization of the receptor through extensive cross-linking leading to the inhibition of hematopoietic tumor cell growth $[2,16]$. In a syngeneic model, R17 208 prolonged the survival of AKR/J mice bearing SL-2 leukemic cells. Although acute toxicity associated with antibody administration was minimal in these animals, the $\operatorname{IgM}$ decreased the number of bone marrow CFU-e (colony forming units-erythroid) and increased progenitor cellularity in the spleen resulting in splenomegaly. Toxicity in mice has also been observed with the use of a non-neutralizing mouse/human chimeric IgG1 antibody targeting murine TfR1 (anti-TfR ${ }^{\mathrm{D}}$ ) [17,18]. In this case, treated mice showed acute clinical signs that were completely reversible within hours and decreased circulating reticulocytes attributed to the Fc effector functions (ADCC and/or CDC) of the antibody.

Another example is the murine anti-human TfR1 $\operatorname{IgA}$ antibody $42 / 6$ that is cytotoxic to malignant hematopoietic cells. 42/6 blocks Tf binding through steric hindrance and downregulates surface TfR1, decreasing iron uptake. $42 / 6$ was shown to be cytotoxic to both normal and malignant myeloid cells at similar levels in vitro [19]. Importantly, a Phase I clinical trial was conducted in patients with various advanced refractory cancers [20]. Although some patients with hematopoietic malignancies demonstrated a mixed antitumor response, no partial or complete remissions were observed. Treatment induced slight myeloid depletion; however, this depletion was not significant. Efficacy may have been hampered by the fast clearance rate of murine IgA and the fact that an anti-murine IgA antibody response was detected in patients. Other murine antibodies targeting human TfR1 such as A24 (IgG2b), E2.3 (IgG1), and A27.15 (IgG1) have 
shown preclinical antitumor activity $[2,21]$. However, versions of antibodies containing human constant regions would maximize the probability of clinical success being better immunologically tolerated and also exhibiting optimal effector functions against tumors in humans.

The ch128.1Av chimeric fusion protein mentioned above has the advantage of having human constant regions. This molecule was originally designed as a delivery vehicle, but also has direct cytotoxic activity against certain malignant cells in vitro through the disruption of the TfR1 cycling, degradation of the receptor, and ultimately iron starvation [7,22]. Importantly, ch128.1Av is not cytotoxic to normal hematopoietic stem cells even when conjugated to the plant toxin saporin due to their lack of TfR1 expression [10]. This observation suggests that this important cell population would be spared in therapies targeting TfR1 and also suggests that this strategy can be used for ex vivo approaches such as purging of cancer cells in autologous bone marrow and stem cell transplantation protocols. The parental antibody without avidin (ch128.1) also exhibits cytotoxicity in vitro, although less than that of ch128.1Av [7]. However, in xenograft models of human multiple myeloma (MM) and non-Hodgkin lymphoma (NHL) ch128.1 has demonstrated significant anticancer efficacy, which in the case of the MM models is greater than that of the fusion protein [23,24]. Interestingly, in vivo antitumor activity is observed even against malignant cells that are not sensitive to the cytotoxic activity of the antibody in vitro. Importantly, this antibody triggers Fc effector functions, which may play a role in its antitumor activity in vivo [25]. Antibodies of complete human nature targeting TfR1 have also been developed including a human $s c F v$ targeting TfR1, which antagonizes malignant hematopoietic cell growth

\section{References}

1 Daniels TR, Bernabeu E, Rodríguez JA et al. The transferrin receptor and the targeted delivery of therapeutic agents against cancer. Biochim. Biophys. Acta 1820(3), 291-317 (2012).

2 Daniels TR, Delgado T, Rodriguez JA, Helguera G, Penichet ML. The transferrin receptor part I: biology and targeting with cytotoxic antibodies for the treatment of cancer. Clin. Immunol. 121(2), 144-158 (2006).

3 Kenneth NS, Mudie S, Naron S, Rocha S. TfR1 interacts with the IKK complex and is involved in IKK-NF-kappaB signalling. Biochem. J. 449(1), 275-284 (2013).

4 Jeong SM, Hwang S, Seong RH. Transferrin receptor regulates pancreatic cancer growth by modulating mitochondrial respiration and ROS generation. Biochem. Biophys. Res. Commun. 471(3), 373-379 (2016). in vitro [26] and a fully human IgG antibody that has shown antitumor efficacy in xenograft models of human oral squamous cell carcinoma in mice [27].

For several years, TfR1 has been studied as a target for antibody-based cancer therapy. Early excitement was dampened due to a combination of factors, including immunogenicity of murine antibodies in humans, lack of efficacy, and/or safety concerns. However, genetic engineering of antibodies including the development of those with human constant regions and the advancement in the formulation of the delivered cargo have greatly facilitated the resurgence in the excitement for the use of TfR1 as a target for antibody-based cancer therapy for both purposes: as a delivery vehicle and as a direct cytotoxic agent. Importantly, studies in animal models and initial clinical trials suggest that certain antibody-based cancer therapeutics will be effective in humans. However, given the broad expression of TfR1 on normal cells, toxicity must always be carefully monitored. Obviously, the ultimate use of these strategies for cancer therapy will depend on the results of clinical trials.

\section{Financial \& competing interests disclosure}

Our research on antibodies targeting TfR1 has been supported in part by the NIH grants K01CA86915, K01CA138559, R01CA107023, R01CA168482, R01CA196266, P30AI028697, and P30CA016042. ML Penichet is a shareholder of Klyss Biotech, Inc. The Regents of the University of California are in discussions with Klyss Biotech, Inc. to license Penichet's technology to this firm. The authors have no other relevant affiliations or financial involvement with any organization or entity with a financial interest in or financial conflict with the subject matter or materials discussed in the manuscript apart from those disclosed.

No writing assistance was utilized in the production of this manuscript.
5

Bakema JE, Van Egmond M. Fc receptor-dependent mechanisms of monoclonal antibody therapy of cancer. Curr. Top. Microbiol. Immunol. 382, 373-392 (2014).

$6 \quad \mathrm{Ng}$ PP, Dela Cruz JS, Sorour DN et al. An anti-transferrin receptor-avidin fusion protein exhibits both strong proapoptotic activity and the ability to deliver various molecules into cancer cells. Proc. Natl Acad. Sci. USA 99(16), 10706-10711 (2002).

$7 \quad \mathrm{Ng}$ PP, Helguera G, Daniels TR et al. Molecular events contributing to cell death in malignant human hematopoietic cells elicited by an IgG3-avidin fusion protein targeting the transferrin receptor. Blood 108(8), 2745-2754 (2006).

8 Rodriguez JA, Helguera G, Daniels TR et al. Binding specificity and internalization properties of an antibodyavidin fusion protein targeting the human transferrin receptor. J. Control. Rel. 124(1-2), 35-42 (2007). 
9 Daniels TR, Ng PP, Delgado T et al. Conjugation of an anti transferrin receptor IgG3-avidin fusion protein with biotinylated saporin results in significant enhancement of its cytotoxicity against malignant hematopoietic cells. Mol. Cancer Ther. 6(11), 2995-3008 (2007).

10 Daniels-Wells TR, Helguera G, Rodriguez JA et al. Insights into the mechanism of cell death induced by saporin delivered into cancer cells by an antibody fusion protein targeting the transferrin receptor 1. Toxicol. In vitro 27(1), 220-231 (2013).

11 Leoh LS, Morizono K, Kershaw KM, Chen IS, Penichet ML, Daniels-Wells TR. Gene delivery in malignant B cells using the combination of lentiviruses conjugated to anti-transferrin receptor antibodies and an immunoglobulin promoter. $J$. Gene Med. 16(1-2), 11-27 (2014).

12 Sugyo A, Tsuji AB, Sudo H et al. Evaluation of efficacy of radioimmunotherapy with 90Y-labeled fully human anti-transferrin receptor monoclonal antibody in pancreatic cancer mouse models. PLoS ONE 10(4), e0123761 (2015).

13 Ghetie V, Vitetta E. Immunotoxins in the therapy of cancer: from bench to clinic. Pharmacol. Ther. 63(3), 209-234 (1994).

14 Laske DW, Muraszko KM, Oldfield EH et al. Intraventricular immunotoxin therapy for leptomeningeal neoplasia. Neurosurgery 41(5), 1039-1049; discussion 1049-1051 (1997).

15 Senzer N, Nemunaitis J, Nemunaitis D et al. PhaseI study of a systemically delivered $\mathrm{p} 53$ nanoparticle in advanced solid tumors. Mol. Ther. 21(5), 1096-1103 (2013).

16 Sauvage CA, Mendelsohn JC, Lesley JF, Trowbridge IS. Effects of monoclonal antibodies that block transferrin receptor function on the in vivo growth of a syngeneic murine leukemia. Cancer Res. 47(3), 747-753 (1987).

17 Couch JA, Yu YJ, Zhang Y et al. Addressing safety liabilities of TfR bispecific antibodies that cross the blood-brain barrier. Sci. Transl. Med. 5(183), 183ra157, 181-112 (2013).

18 Yu YJ, Zhang Y, Kenrick M et al. Boosting brain uptake of a therapeutic antibody by reducing its affinity for a transcytosis target. Sci. Transl. Med. 3(84), 84 ra44 (2011).

19 Taetle R, Honeysett JM, Trowbridge I. Effects of antitransferrin receptor antibodies on growth of normal and malignant myeloid cells. Int. J. Cancer 32 (3), 343-349 (1983).

20 Brooks D, Taylor C, Dos Santos B et al. Phase Ia trial of murine immunoglobulin A antitransferrin receptor antibody 42/6. Clin. Cancer. Res. 1(11), 1259-1265 (1995).

21 Callens C, Moura IC, Lepelletier Y et al. Recent advances in adult T-cell leukemia therapy: focus on a new anti-transferrin receptor monoclonal antibody. Leukemia 22 (1), 42-48 (2008).

22 Rodriguez JA, Luria-Perez R, Lopez-Valdes HE et al. Lethal iron deprivation induced by non-neutralizing antibodies targeting transferrin receptor 1 in malignant B cells. Leuk. Lymphoma 52(11), 2169-2178 (2011).

23 Daniels TR, Ortiz-Sanchez E, Luria-Perez R et al. An antibody-based multifaceted approach targeting the human transferrin receptor for the treatment of B-cell malignancies. J. Immunother. 34(6), 500-508 (2011).

24 Daniels-Wells TR, Widney DP, Leoh LS, Martinez-Maza $\mathrm{O}$, Penichet ML. Efficacy of an anti-transferrin receptor 1 antibody against AIDS-related non-Hodgkin lymphoma: a brief communication. J. Immunother. 38(8), 307-310 (2015).

25 Leoh LS, Daniels-Wells TR, Martinez-Maza O, Penichet ML. Insights into the effector functions of human IgG3 in the context of an antibody targeting transferrin receptor 1. Mol. Immunol. 67(2 Pt B), 407-415 (2015).

26 Crepin R, Goenaga AL, Jullienne B et al. Development of human single-chain antibodies to the transferrin receptor that effectively antagonize the growth of leukemias and lymphomas. Cancer Res. 70(13), 5497-5506 (2010).

27 Nagai K, Nakahata S, Shimosaki S et al. Development of a complete human anti-human transferrin receptor $\mathrm{C}$ antibody as a novel marker of oral dysplasia and oral cancer. Cancer Med. 3(4), 1085-1099 (2014). 